\title{
Commentary on MEDEA: A DSGE model for the Spanish economy
}

\author{
Filippo Ferroni
}

(C) The Author(s) 2010. This article is published with open access at Springerlink.com

The paper provides a comprehensive description of the Spanish economy. The model (MEDEA) is built on recent developments of medium scale DSGE models, and it has been enriched by adding domestic and foreign sectors, several frictions on the real and nominal side of the economy. Moreover, MEDEA features important departures from the paradigm of DSGE models to closely mimic the evolution of the Spanish economy in the past 30 years.

The main novelty with respect to the existing literature is the introduction of three sources of growth: neutral technological progress, investment-specific technological progress, and population growth. Immigration flows heavily influenced the Spanish economy in the recent past, and the attempt to model it is clearly an interesting idea. On top of that, the authors try to define a monetary policy rule that replicates closely the Spanish role in Europe Area and in the World economy, and they attempt to provide a better characterization of fiscal policy instruments. Finally, despite of its complexity the model description is very clear and the notation is easy to follow.

MEDEA is estimated via Bayesian methods. The authors' enterprise to estimate the model is fully aware of potential problems that likelihood based estimation of DSGE might carry, namely weak parameters identification and potential parameters instability. First, to reduce identification problems just a subset of structural parameters is estimated (47 parameters out of 59), and flat prior are used to let the data reveals fully the information about structural parameters. The model is treated analytically in such a way that it is feasible to implement II order approximation of the model solution; eventually, likelihood based estimation on II order approximation might be performed

This commentary refers to the article available at: doi:10.1007/s13209-009-0011-x.

\section{F. Ferroni $(\bowtie)$}

Banque De France, 31 rue Croix des Petits Champs, 75001 Paris, France

e-mail: filippo.ferroni@banque-france.fr 
to introduce more curvature to the likelihood and to better identify parameters. Unfortunately, current computer powers make this option unfeasible. Second, estimation of rolling windows on different subsamples are performed to check parameters stability.

Overall, I think that the model is well designed for the Spanish economy, and the estimation procedure is conducted with a lot of care. Following a common tendency among policy making institutions, MEDEA is meant to be used for multitasking exercises, such as forecasting, policy evaluation and counterfactual exercise. I am a bit skeptical about this point; I believe that the 'loss' function behind a forecasting exercise is pretty different from that of counterfactual experiments. In the former we look for models and specifications that maximize some out-of-sample accuracy, in the latter we look for models and specifications able to tell 'reasonable' economic stories. Not necessary the two approaches go to the same direction.

In this discussion, I organize specific doubts about the paper, in particular about the introduction of population evolution, the potential identification problems for some parameter and the specification of the fiscal policy rule.

\section{Population evolution and model based data transformations}

As mentioned, introducing the population growth in the model is a clever extension given the recent past immigration flows in Spain. In MEDEA the population evolution is postulated to follow a random walk. However, looking at the Spanish population data $^{1}$ (Fig. 1), I am not convinced that the series displays a stochastic pattern.

If we run a unit root test on this series, we get a Dickey Fuller statistic of -39.0899 . At $5 \%$ significance level, the critical value is -7.9 . Thus, we strongly reject the null hypothesis of unit root. Moreover, we all know that Dickey Fuller test is strongly bias toward accepting the unit root hypothesis. So, it seems that the hypothesis of unit root is not supported by the data. Does this have an impact on the MEDEA structural parameter estimates other than the population growth?

While with limited information methods mispecification in one equation is not going to affect much other parameter estimates, with full information methods mispecification in one equation spreads all over the system making our estimates unreliable. In a seminal paper, Cogley (2001) shows that a wrong trend specification leads to strong bias in parameter estimates with likelihood based methods. Even when the reduced form of the cyclical component is correctly specified, trend mispecification is likely to results in inconsistent estimates of 'deep' parameters. Even though the concept of bias makes little sense in a Bayesian context being a property of classical estimators, there is still a concern about the potential posterior distribution of the parameters that might be very different form those obtained by a linear trend specification. Distinct posterior distributions imply dramatically different conclusions of the model (i.e. in terms of IR, variance decompositions etc...). If this is the case, then a Bayes factor comparison should reveal which of the two specification (linear trend vs. stochastic one) is more likely.

\footnotetext{
${ }^{1}$ Data has been gently provided by the authors.
} 


\section{LEVELS}
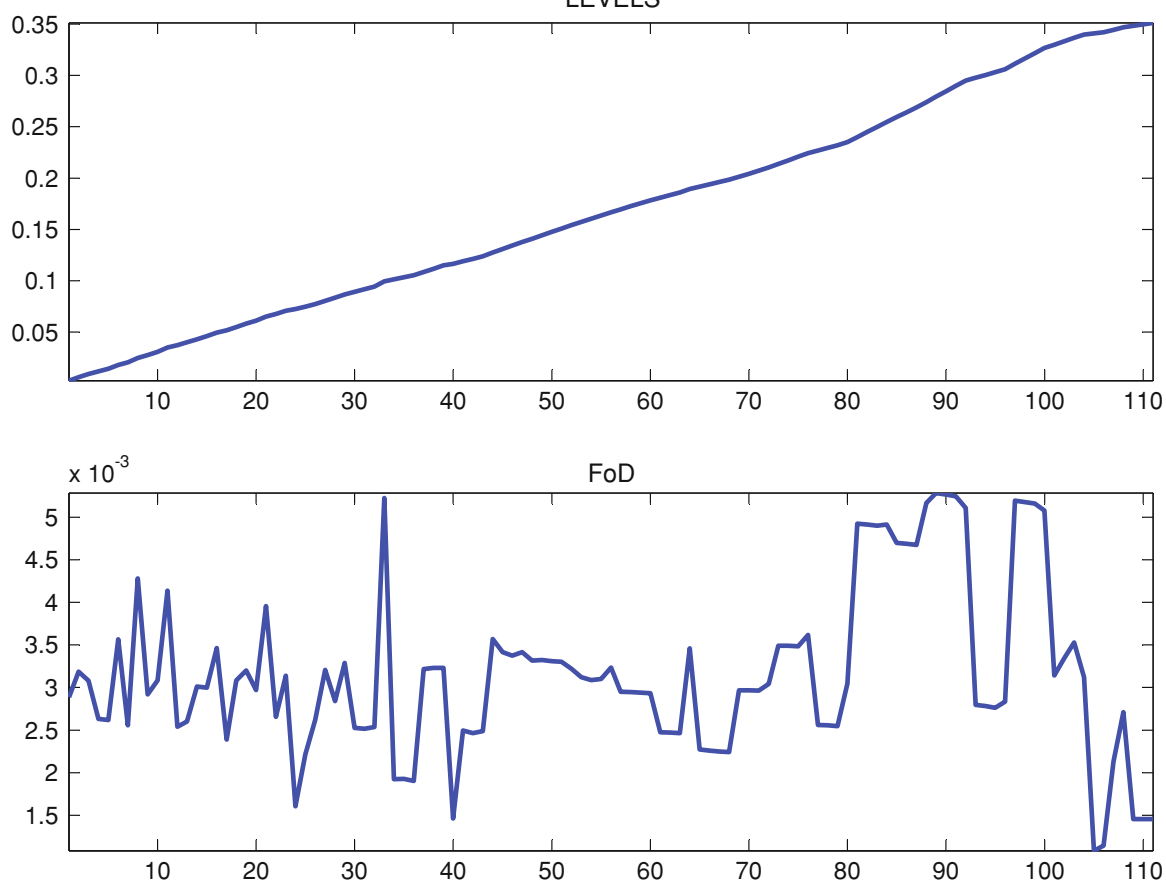

Fig. 1 Population evolution in Spain from June 1980 to December 2007

I have broad concerns about the employment of model based transformations. The three unit roots postulated in the model allow the authors to take first difference on the data. I am not fully satisfied with such an approach. First, this assumption implies that 'great ratios' are purely cyclical in the model, whereas the data counterparts display long run movements. In particular, consumption and output grow at the same rate in the model, and thus the ratio between the two should be stationary.

Looking at Fig. 2, the ratio between consumption and output do not look stationary. Second, the interpretation of the cycles might be misleading with a first difference filter: if we define cycles as fluctuations with periodicity among 8-32 quarters, taking first difference on the data does not extract such fluctuations. Third, with small sample sizes variability and persistence of a series is generally mismeasured with single filtering; i.e. volatilities of first difference data simulated from a DSGE model with stochastic growth are statistically different form the 'true' cycles ones, see Canova and Ferroni (2008). Fourth, trend parameters might be unstable along the sample; long run movements of the data can display breaks of various form.

Recently, several papers are trying to tackle these issues. With limited information approach, Fukac and Pagan (2007) propose method to deal with the treatment of trend in DSGE estimations. Gorodnichenko and Ng (2007) extend their analysis and propose a robust approach exploiting all the cross-equations restrictions of the DSGE model. While their analysis is confined to data that share cointegrated patters and that do not display breaks, Ferroni (2009) shows how to estimate structural parameters 
Fig. 2 Ratio between consumption and output June 1980- December 2007

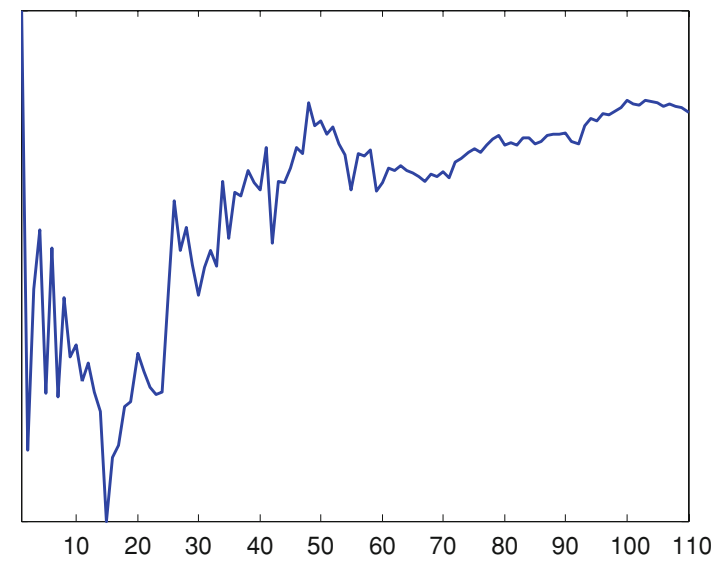

of DSGE using a flexible trend specification, where times series do not necessary display similar upward trends (such as GDP and M2, see Mehra (1997)) or structural brakes along the sample, see Perron and Wada (forthcoming). Canova and Ferroni (2008) propose an implementable method that allows to estimate 'deep' parameters exploiting all the information proceeding from different data transformations, and to consistently account for the mismeasurement of the cyclical component.

\section{Identification issues}

I suspect that the growth rate of technology and of investment specific shock, $\Lambda_{A}$ and $\Lambda_{\mu}$, are not jointly identifiable by construction. For the sake of the argument lets focus only on the real part of the model. The loglinearized solution of MEDEA can be represented in a state space representation, i.e.

$$
\begin{aligned}
\Delta \ln y_{t}^{\text {real }, O} & =\ln \widetilde{z}+\gamma^{L}+\widehat{\gamma}_{t}^{L}+O \Delta y_{t}^{\dagger} \\
y_{t+1}^{\dagger} & =W(\Psi) y_{t}^{\dagger}+\epsilon_{t+1}
\end{aligned}
$$

where $y_{t}^{\text {real, } O}$ are the real observable variables, $y_{t}^{\dagger}$ is the vector of model based variables that evolves with a VAR representation and $O$ is a selection matrix that picks out of $y_{t}^{\dagger}$ those variable that are expressed in real terms and are observable. $\gamma^{L}$ and $\widehat{\gamma}_{t}^{L}$ are related to the population evolution and $\ln \widetilde{z}=\frac{\Lambda_{A}+\alpha \Lambda_{\mu}}{1-\alpha}$. In the measurement equation we are not able separately identify growth rate of technology and of investment specific shock, $\Lambda_{A}$ and $\Lambda_{\mu}$. Also in the loglinearized equilibrium equations they never appear separately.

The fact that they are not identifiable is clear enough if we look at priors and posteriors distributions, where prior and posterior virtually coincide. I suggest to fix either $\Lambda_{A}$ or $\Lambda_{\mu}$. 


\section{Fiscal policy (feedback) rule}

I have a last comment on the fiscal policy specification. It is assumed that government levy distortionary taxes on household consumption and on household revenue from renting capital and from labor, $\tau^{c}, \tau^{k}$ and $\tau^{l}$ respectively. Moreover, an exogenous government spending process is postulated.

In the introduction, the authors put emphasis on the fiscal policy treatment. So, I was expecting some form of tax policy feedback rule, where taxes respond to cyclical condition of the economy, like employment or GDP (see Jones (2002)). Unfortunately, there is no room for countercyclical behavior for fiscal policy. A loose tax rule on transfers is postulated, which responds only to debt variations. It would interesting to introduce some sort of automatic response of transfers to cyclical conditions; for example, to mimic the Spanish subsidy for unemployment transfers could respond to employment supply variations, i.e.

$$
\frac{T_{t}}{y_{t}^{d}}=T_{0}-T_{1}\left(\widetilde{b}_{t}-\overline{\widetilde{b}}_{t}\right)+T_{2} l_{t}^{s}
$$

My guess is that the estimate of $T_{2}$ should be negative. Thus, we should observe a countercyclical behavior of transfers. Then, one could test different scenarios of $T_{2}$ and measure the impact on the volatility of the macro variables.

Open Access This article is distributed under the terms of the Creative Commons Attribution Noncommercial License which permits any noncommercial use, distribution, and reproduction in any medium, provided the original author(s) and source are credited.

\section{References}

Burriel P, Fernández-Villaverde J, Rubio-Ramírez JF (2010) MEDEA: a DSGE model for the Spanish economy. FEDEA Working Paper 2009-17. SERIEs 1:175-243

Canova F, Ferroni F (2008) Multiple fitlering device for the estimation of cyclcical DSGE models. Working paper, UPF working paper series

Cogley T (2001) Estimating and testing rational expectations models when the trend specification is uncertain. J Econ Dyn Control 25(10):1485-1525

Ferroni F (2009) Trend agnostic one step estimation of DSGE models., Mimeo

Fukac M, Pagan A (2007) Limited information estimation and evaluation of DSGE models, economics working papers 6 , NCER

Gorodnichenko Y, Ng S (2007) Estimation of DSGE models when the data are persistent, NBER working papers, National Bureau of Economic Research

Jones JB (2002) Has fiscal policy helped stabilize the postwar US economy? J Monet Econ 49(4):709-746

Mehra YP (1997) A review of the recent behavior of M2 demand. Econ Quart (Sum) 83:27-44

Perron P, Wada T (2010) Let's take a break: trends and cycles in the US real GDP. J Monet Econ (forthcoming) 